\title{
NANOCRYSTAL HYBRIDIZED WHITE LIGHT SOURCES INTEGRATED ON NEAR UV LEDS
}

\author{
S. NIZAMOGLU, H. V. DEMIR \\ Department of Physics, Department of Electrical and Electronics Engineering, \\ Nanotechnology Research Center, Bilkent University \\ Bilkent, 06800 Ankara, Turkey
}

\begin{abstract}
We report on $\mathrm{CdSe} / \mathrm{ZnS}$ core-shell nanocrystal (NC) based white light sources integrated on near-UV InGaN/GaN light emitting diodes (LEDs). We present the design, epitaxial growth, fabrication, integration and characterization of such hybrid NC-LEDs that incorporate the dual combinations of cyan and yellow nanocrystals $\left(\lambda_{\mathrm{PL}}=500-580 \mathrm{~nm}\right)$ and the trio combinations of cyan, green, and red nanocrystals $\left(\lambda_{\mathrm{PL}}=500-540-620 \mathrm{~nm}\right)$.
\end{abstract}

\section{Introduction}

Recently research and development in white light emitting technology has resulted in many practical applications, e.g., in automotive industry [1]. Because of the wide scale demand for white light emitting diodes (WLED), different techniques have been investigated including multi-chip WLEDs, monolithic WLEDs, and color-conversion WLEDs. Among these techniques, the wavelength conversion method based on phosphor is the most widely used technique, which has been also commercialized [2]. However, since the phosphor has a wide fixed photoemission spectrum, the optical property of the generated light is not easily adjustable.

Recently, white light generation has been achieved using a nano-hybrid device approach that relies on the use of nanocrystals (NCs) in our research group and others [3-7]. Furthermore, it has been shown that tunable white light can be accomplished using combinations of nanocrystals in our group [6,7]. However, previous research work has relied only on the use of visible LEDs. Notably, experimental work on white light generation with nanocrystal hybridization on a near UV source has not previously been demonstrated.

For the first time, in this work we present the design, growth, fabrication, integration and characterization of hybrid $\mathrm{CdSe} / \mathrm{ZnS}$ core-shell nanocrystals based white light sources integrated on near-UV InGaN/GaN LEDs. Such UV-pumped nanocrystal white light sources allows for the flexible choice of combinations of nanocrystals to contribute to white light generation with their photoluminescence in the visible range. Here we demonstrate the dual combination of cyan and yellow NCs $\left(\lambda_{\mathrm{PL}}=500-580 \mathrm{~nm}\right)$ and the trio combination of cyan, green and red NCs $\left(\lambda_{\mathrm{PL}}=500-540-620 \mathrm{~nm}\right)$ hybridized on a $\mathrm{n}-\mathrm{UV}$ LED 
$\left(\lambda_{\mathrm{EL}}=383 \mathrm{~nm}\right)$ to yield tristimulus coordinates of $(\mathrm{x}, \mathrm{y})=(0.30,0.28)$ and $(\mathrm{x}, \mathrm{y})=(0.27,0.46)$, respectively.

\section{Operating principle and design}

The operating principle of such a hybrid NC-LED relies on the hybrid use of the LED as the pump light source and the integrated NC films as the photoluminescent layers. When electrically driven, the UV LED optically pumps the NCs, and the photoluminescence of the NCs generates the white light. For white light generation, we use $\mathrm{CdSe} / \mathrm{ZnS}$ core-shell NCs that give photoluminescence in the visible.

The narrow photoluminescence (PL) spectrum of these NCs are widely tunable across the visible spectrum to adjust white light parameters, whereas the conventional yellow phosphorus has a fixed PL spectrum. By using the size effect, these NCs can fully cover the visible spectrum from blue to red as shown in Fig. 1a-d. Additionally, the small overlap of the NC emission and absorption spectra provides the ability to tune the white light spectrum conveniently. We carefully design and implement hybrid device parameters such as the order and thickness of the NC films and type and density of NCs as necessary to generate white light with the desired characteristics.

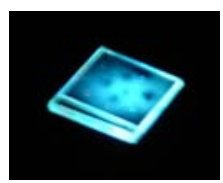

a)

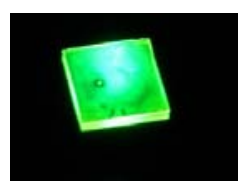

b)

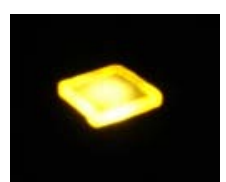

c)

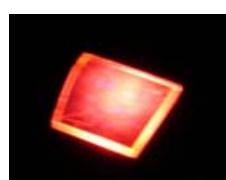

d)

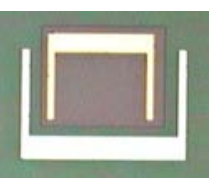

e)

Figure 1. Photoluminescence from (a) our blue NC film, (b) our green NC film (c) our yellow NC film (d) our red NC; (e) a top-view micrograph of our fabricated near-UV InGaN/GaN LED.

For the hybridization platform, we use near-UV InGaN/GaN LEDs that we design, grow, and fabricate. For their epitaxial growth, we use Aixtron RF200/4 RF-S MOCVD system at Bilkent Nanotechnology Research Center. On sapphire we grow in the following order: $14 \mathrm{~nm} \mathrm{GaN}$ nucleation layer, $200 \mathrm{~nm} \mathrm{GaN}$ buffer layer, $690 \mathrm{~nm}$ Si doped n-type contact layer, five 2-3 nm InGaN wells and $\mathrm{GaN}$ barriers (at $720{ }^{\circ} \mathrm{C}$ ), $50 \mathrm{~nm} \mathrm{Mg}$ doped p-type $\mathrm{AlGaN}$, and finally $120 \mathrm{~nm}$ $\mathrm{Mg}$ doped p-type GaN layer. We use standard semiconductor processing for the fabrication that includes photolithography, thermal evaporator (metallization), reactive ion etch (RIE), and rapid thermal annealing. For p-contact, we use $\mathrm{Ni} / \mathrm{Au}(15 / 100 \mathrm{~nm})$ and anneal at $700{ }^{\circ} \mathrm{C}$ for $30 \mathrm{~s}$. For the n-contact, we etch with RIE down to $940 \mathrm{~nm}$, lay down $\mathrm{Ti} / \mathrm{Al}(10 / 200 \mathrm{~nm})$ and anneal at $600{ }^{\circ} \mathrm{C}$ for 1 min under $\mathrm{N}_{2}$ purge. For hybridization, we integrate various types of NCs on 
one of our fabricated LEDs, shown in Fig. 1e.

\section{Characterization}

We use four types of $\mathrm{CdSe} / \mathrm{ZnS}$ core-shell NCs with crystal diameters of $1.9 \mathrm{~nm}$, $2.4 \mathrm{~nm}, 3.2 \mathrm{~nm}$, and $5.2 \mathrm{~nm}$, and size distributions of $\sim 5 \%$. Their emission colors are cyan, green, yellow and red, with corresponding photoluminescence peaks at $500 \mathrm{~nm}, 540 \mathrm{~nm}, 580 \mathrm{~nm}$ and $620 \mathrm{~nm}$, respectively. We use n-UV LEDs with their electroluminescence peak wavelength at $383 \mathrm{~nm}$.

Hybridizing dual combinations of cyan and yellow NCs $\left(\lambda_{\mathrm{PL}}=500 \mathrm{~nm}\right.$ and $580 \mathrm{~nm}$ ) with the respective order and carefully designed device parameters, we obtain the emission spectra at various levels of current injection shown in Fig. 2a, resulting in $(\mathrm{x}, \mathrm{y})=(0.36,0.48), \mathrm{T}_{\mathrm{c}}=4893$, and $\mathrm{R}_{\mathrm{a}}=49.70$. Hybridizing trio combinations of cyan, green, and red $\mathrm{NCs}\left(\lambda_{\mathrm{PL}}=500 \mathrm{~nm}, 540 \mathrm{~nm}\right.$ and $620 \mathrm{~nm}$ ), again with the respective order and right device parameters, we obtain the emission spectra at various current injection levels shown in Fig. 2b, leading to $(\mathrm{x}, \mathrm{y})=(0.27,0.46), \mathrm{T}_{\mathrm{c}}=7187$, and $\mathrm{R}_{\mathrm{a}}=49.88$. These operating points yield near white light, as shown on the C.I.E. Chromaticity Diagram (1931) in Fig. 3 [2,8].

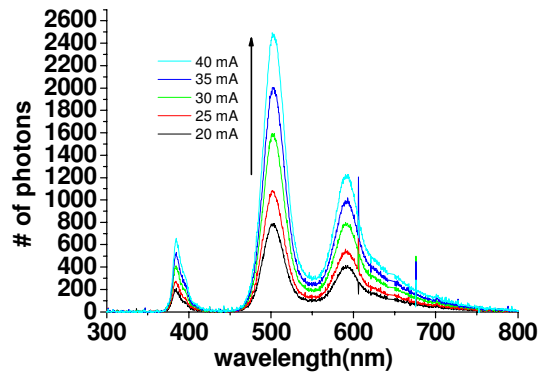

a)

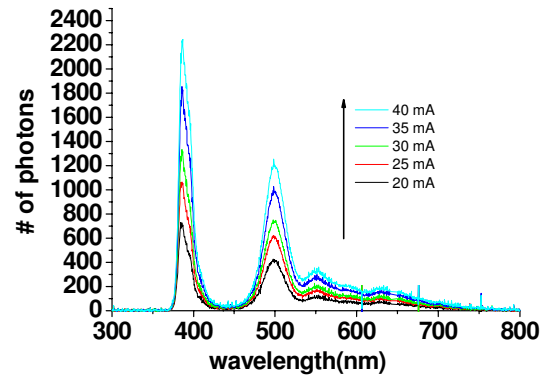

b)

Figure 2. Emission spectra of our hybrid NC-LEDs at various injection currents at room temperature for (a) the dual combination of cyan and yellow NCs, and (b) the trio combination of cyan, green and red NCs.

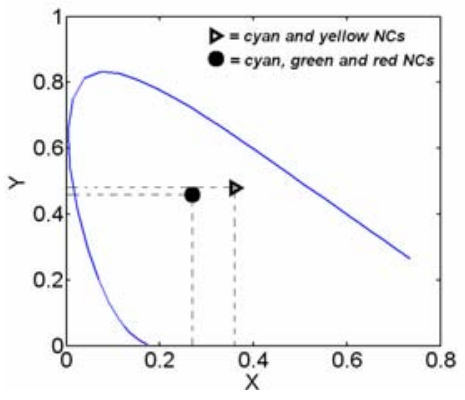

Figure 3. Tristimulus coordinates of our hybrid NC-LEDs on C.I.E. Chromaticity Diagram (1931). 


\section{Conclusion}

We present the design, growth, fabrication, integration and characterization of hybrid dual- and trio-combination nanocrystal LEDs for white light generation. For the first time, we demonstrate the use of near UV LED as for the hybridization platform. Based on our experimental work, we believe that this hybrid approach holds strong promise for future solid state lighting and displays with their highly adjustable optical properties.

\section{Acknowledgments}

This work is supported by a Marie Curie European Reintegration Grant MOON 021391 and EU-PHOREMOST Network of Excellence 511616 within the 6th European Community Framework Program and TUBITAK under the Project Nos. 104E114, 106E020, 105E065, and 105E066. Authors also acknowledge additional support from Turkish Academy of Sciences Distinguished Young Scientist Award Program and TUBITAK Fellowship.

\section{References}

1. M.Arik, J.Petroskf, S.Weavery, in: Inter Society Conference on Thermal Phenomena (2002), pp. 112-120.

2. E.F.Schubert, Light-Emitting Diodes (Cambridge University Press, 2006).

3. H.Chen, D.Yeh, C.Lu, C.Huang, W.Shiao, J.Huang, C.C.Yang, I.Liu, W.Su, IEEE Photon. Technol. Lett. 18, 1430 (2006).

4. H.Chen, C.Hsu, H.Hong, IEEE Photon. Technol. Lett. 18, 193 (2006).

5. M.A.Petruska, D.D.Koleske, M.H.Crawford, V.I.Klimov, Nano Lett. 6, 1396 (2006).

6. S.Nizamoglu, T.Ozel, E.Sari, H.V.Demir, in: IEEE COMMAD Conference on Optoelectronic and Microelectronic Materials and Devices (Perth, Australia, 6-8 Dec. 2006), pp. W0-A5.

7. S.Nizamoglu, T.Ozel, E.Sari, H.V.Demir, to appear in Nanotechnology (2006).

8. C.H.Chen, S.J.Chang, Y.K.Su, J.K.Sheu, J.F.Chen, C.H.Kuo, Y.C.Lin, IEEE Photon. Technol. Lett. 14, 908 (2002). 